\title{
A novel 'Batman' scrotectomy technique for the management of scrotal lymphoedema following treatment for penile cancer
}

\author{
Hussain M. Alnajjar ${ }^{1}$, Fabio Castiglione ${ }^{1}$, Kamran Ahmed ${ }^{1}$, Aiman Haider ${ }^{2}$, Raj Nigam ${ }^{1}$, Asif Muneer ${ }^{1,3,4}$ \\ ${ }^{1}$ Department of Urology, ${ }^{2}$ Department of Pathology, University College London Hospitals NHS Trust, London, UK; ${ }^{3}$ NIHR Biomedical Research \\ Centre University College London Hospitals, London, UK; ${ }^{4}$ Division of Surgery and Interventional Science, UCL, London, UK \\ Contributions: (I) Conception and design: HM Alnajjar, A Muneer; (II) Administrative support: None; (III) Provision of study materials or patients: \\ HM Alnajjar, A Muneer; (IV) Collection and assembly of data: HM Alnajjar, F Castiglione, A Muneer; (V) Data analysis and interpretation: HM \\ Alnajjar, A Muneer; (VI) Manuscript writing: All authors; (VII) Final approval of manuscript: All authors. \\ Correspondence to: Mr Asif Muneer, MD, FRCS(Urol). Consultant Urological Surgeon and Andrologist, University College London Hospitals NHS \\ Trust, London, UK. Email: asif.muneer@nhs.net.
}

\begin{abstract}
Background: To describe a novel 'Batman' scrotectomy technique and present our single centre experience in the management of secondary scrotal lymphoedema in penile cancer patients.

Methods: A retrospective review of the medical records of penile cancer patients with extensive and bothersome penoscrotal lymphoedema failing conservative therapy between 2013 and 2018. We analysed patients' demographics, pre-operative disease stage, post-operative outcomes and complications.

Results: Seven patients with a history of penile cancer and problematic scrotal lymphoedema were managed using a novel 'Batman' scrotectomy technique. The mean age was 56.4 (range, 28-71) years. The mean inpatient stay was 4.1 (range, $2-7$ ) days. Two patients $(28.6 \%$ ) were found to have incidental metastatic squamous cell carcinoma (SCC) in the scrotal skin on histological analysis. One patient developed superficial wound dehiscence (Clavien-Dindo grade II) and two patients had mild post-operative residual penile lymphoedema. Following a mean follow-up period of 19 months, 2 patients died due to metastatic penile cancer. One patient developed skin metastases in his thigh and perineum. All of the patients reported a good cosmetic and functional outcome on post-operative review.

Conclusions: Genital lymphoedema is an uncommon side-effect of penile cancer treatment. In severe cases where patients fail conservative treatments, surgical intervention using this technique is effective and feasible.
\end{abstract}

Keywords: Genital lymphoedema; scrotectomy; penile cancer; inguinal lymphadenectomy

Submitted Apr 28, 2019. Accepted for publication Aug 14, 2019.

doi: $10.21037 /$ tau.2019.09.04

View this article at: http://dx.doi.org/10.21037/tau.2019.09.04

\section{Introduction}

Penoscrotal lymphoedema is a problematic long-term side effect of penile cancer surgery and adjuvant treatment such as inguinal and pelvic radiotherapy. Patients can present with debilitating functional, cosmetic and psychological problems which have a significant impact not only on their own quality of life (QOL) but also affects the relationship with the partner. In addition to pain, other complaints such as chronic irritation, maintaining genital hygiene, impaired ambulation, voiding dysfunction, recurrent infections and the inability to have penetrative sexual intercourse have been reported $(1,2)$. Recurrent cellulitis is also common and leads to a loss of tissue elasticity, hyperplasia of the collagenous connective tissue and the development of fibrosis, this perpetuates further swelling with progressive loss of function (3). In some cases, the development of lymphangitis circumscripta (Figure 1) leads to chronic serous discharge from the scrotal skin.

The lymphatic drainage of the penis and scrotum is 


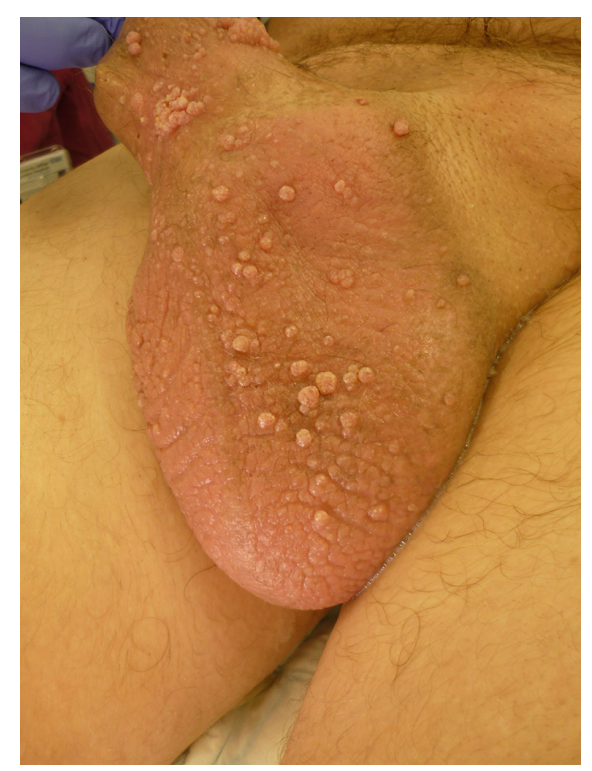

Figure 1 Mild scrotal lymphoedema with lymphangitis circumscripta.

Table 1 Lymphoedema conservative management methods

Conservative lymphoedema management techniques/methods

provided at our dedicated lymphoedema clinic

Supportive hosiery

Manual lymphatic drainage/lymphatic massage

Compression bandaging

Intermittent pneumatic pump therapy

Physical therapy and exercises

Skin care

Limb elevation

Patient education and training

primarily to the inguinal lymph nodes, therefore surgical intervention or radiotherapy in this area leads to disruption of the lymphatics and retention of lymph within the genital subcutaneous tissue and lower limbs. However, the use of dynamic sentinel lymph node biopsy has reduced the incidence of post-operative penoscrotal lymphoedema by reducing the number of patients with clinically impalpable (cN0) inguinal nodes requiring more extensive inguinal lymphadenectomy procedures. However, men with palpable inguinal nodes with metastatic disease still require radical inguinal and potentially pelvic lymphadenectomy together with adjuvant radiotherapy if extracapsular extension of tumour in the lymph nodes is present.

Genital and lower limb lymphoedema management following penile cancer treatment initially involves conservative measures such as life style changes, compression hosiery and massage techniques combined with avoidance of trauma and infection. Long term compressive hosiery and supportive scrotal garments are adequate for the majority of cases (4). However, in severe cases where the size of the scrotum results in poor mobility and a buried penis, leading to a difficult clinical examination of the penis, surgical intervention is often required. So far, a number of surgical techniques including scrotal flaps, skin grafts and lymphangioplasty have been described to manage scrotal lymphoedema (1-3,5-11) although none of these have been used as a standard treatment in penile cancer cases.

The aim of this study is to present a novel 'Batman' scrotectomy technique which allows a safe and effective excision of the scrotal lymphoedema and report the outcomes in our cohort of penile cancer patients. This is the largest reported series of surgically managed scrotal lymphoedema in a cohort of penile cancer patients using this novel technique.

\section{Methods}

The medical records of patients developing bothersome scrotal lymphoedema secondary to cancer treatment between 2013 and 2018 were retrospectively reviewed. Patients with a cancer diagnosis other than penile cancer were excluded from the study. We analysed patients' demographics, pre-operative disease stage, postoperative outcomes, previous episodes of cellulitis and histopathological review.

All patients were initially managed conservatively and were regularly assessed and managed in a dedicated lymphoedema clinic. Their conservative treatment course was dictated as per the hospital's protocol for managing patients with lymphoedema (Table 1).

All patients underwent a scrotectomy technique using a 'Batman' shaped incision (Figure 2) by either of the authors Muneer or Alnajjar. The details of the surgical technique are described below.

\section{Surgical technique}

Following informed consent, intravenous broad-spectrum antibiotics were given. Standard skin preparation and 


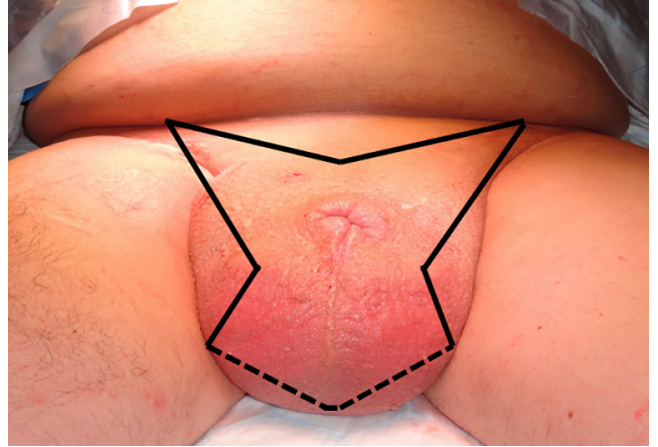

Figure 2 Extensive genital lymphoedema resulting in a buried penis. The "Batman" incision lines, dotted line goes under the scrotum and joins the lateral scrotal folds superiorly.

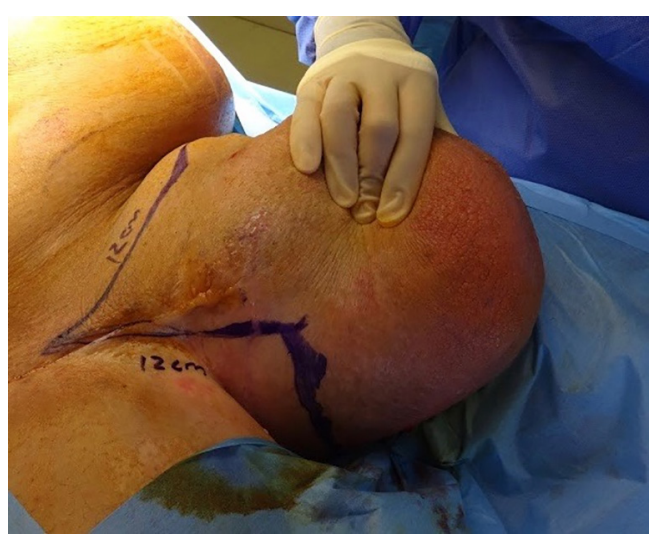

Figure 3 Incision lines are marked bilaterally to the lateral scrotal folds in order to allow one edge to rotate and meet the superior border and the lower edge meets in the midline to create the midline raphe of the neo-scrotum.

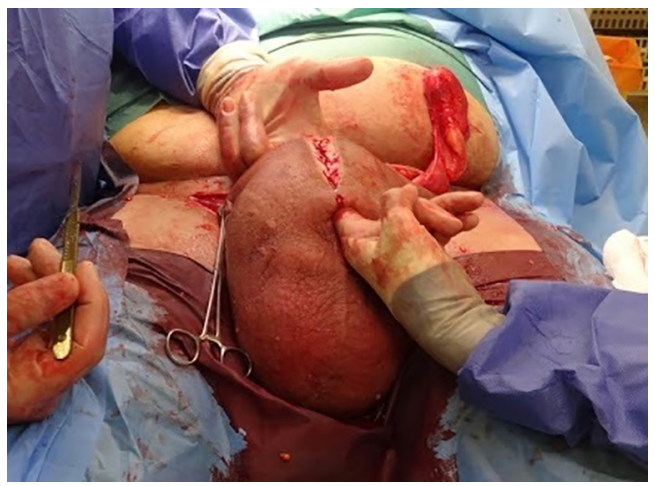

Figure 4 Dorsal skin incision is used to expose the penile shaft. The testes and spermatic cords are isolated and protected.

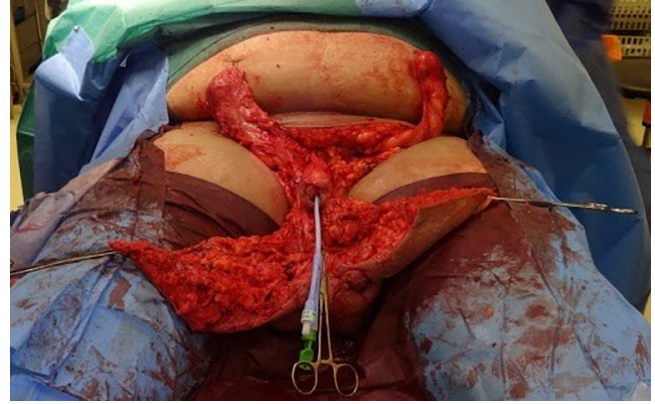

Figure 5 Penile shaft has been exposed. As the spermatic cord is well out of the field, the scrotal skin can be excised without damaging any other vital structures.

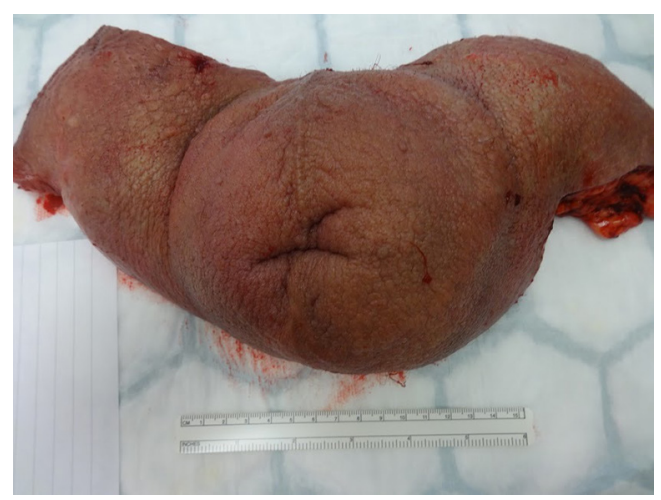

Figure $61.4 \mathrm{~kg}$ of skin and subcutaneous oedematous tissue excised from the penoscrotal and inguinal regions.

draping of the patient was performed with the patient placed in a supine position with the legs abducted (Figure 2). The skin to be excised was marked out (Figures 3 and 4). The skin markings extend laterally such that the preserved lateral scrotal folds would rotate and meet in the midline to create a new raphe. As the lateral skin folds have lymphatic drainage through an alternative pathway, this area is unaffected by inguinal lymphadenectomy. It is essential to excise all of the affected tissue to avoid the risk of recurrence of lymphoedema. The edge of the scrotal flap was measured to ensure that it was half the length of the superior border of the incision such that it can be rotated and sutured to the superior border. Following the incision, the spermatic cord is identified so that the cord structures and testicles were brought out of the operative field (Figure 5). Once the penis was mobilised the scrotum was excised along the skin markings 


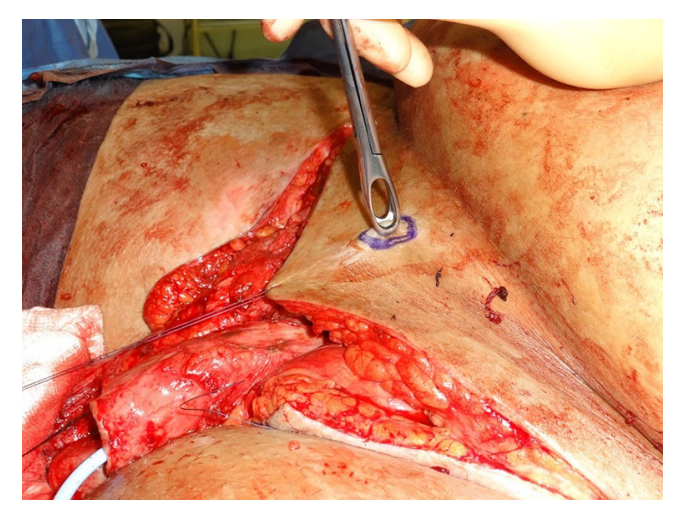

Figure 7 Separate circular incision line is made in the midline, on the suprapubic region as the new location for the penile shaft.

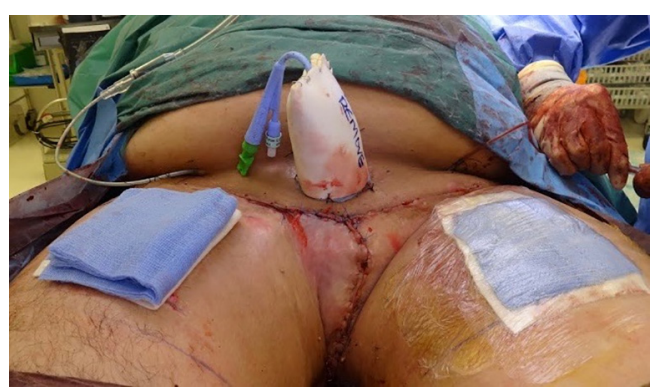

Figure $\mathbf{8}$ Penis is covered with a split-thickness skin graft harvested from the thigh as this patient had undergone radiotherapy. Other options include a full thickness skin graft or a meshed split thickness skin graft. The wound is closed in layers to reduce any dead space and the testes are repositioned in neo-scrotum.

Table 2 Advantages and disadvantages of split-thickness versus fullthickness skin graft for penile reconstruction

\begin{tabular}{ll}
\hline Split-thickness skin graft & Full-thickness skin graft \\
\hline Graft contraction, less flexible & Lower graft contraction, flexible \\
Susceptible to trauma & Lower susceptibility to trauma \\
$\begin{array}{l}\text { Higher discrepancy in skin } \\
\text { pigmentation }\end{array}$ & $\begin{array}{l}\text { Lower discrepancy in skin } \\
\text { pigmentation }\end{array}$ \\
Takes more easily & Takes less easily \\
$\begin{array}{l}\text { Poorer cosmetic result on the } \\
\text { penis }\end{array}$ & $\begin{array}{l}\text { Better cosmetic result for the } \\
\text { penile shaft }\end{array}$ \\
\hline
\end{tabular}

(Figure 6). The testicles were replaced in the neo-scrotum and the tunica vaginalis sutured together followed by approximation of the lateral scrotal folds in the midline. The upper edge of the lateral scrotal folds joined the superior margin of the incision with the lower part being sutured in the midline to create the neo-scrotum. If necessary, the penis was brought out through a new circular opening created above the sutured line (Figure 7). This reduced the number of skin junctions and allowed better post-operative healing. The penile shaft can be covered with either a split skin or full thickness extra-genital skin graft if there is deficiency of penile shaft skin or abnormal skin due to lichen sclerosus (Figure 8). The relative advantages and disadvantages of split-thickness versus full-thickness skin grafts are shown in Table 2. Redivac (close drain system) drains were inserted into each side of the scrotum. The patient was catheterized with post-operative instructions for strict bed rest for 24 hours.

All of the excised tissue was sent for histopathological analysis to confirm the diagnosis and exclude disease recurrence within the scrotal skin.

Patients were encouraged to mobilize on post-operative day 2 and the catheter was removed once the patient was mobile. The drains were removed once drainage was recorded as $<50 \mathrm{~mL}$ on each side over a 24 -hour period. Pre-operative, intra-operative and post-operative parameters were recorded. Post-operative complications, were recorded according to the Clavien-Dindo classification (Table 3).

Where skin grafts were used on the shaft, the indwelling Foley catheter and dressings were removed at between $7-10$ days to ensure that the graft had taken.

\section{Results}

A total of seven patients with scrotal lymphoedema following penile cancer treatment underwent the 'Batman' scrotectomy technique. The mean age of the patients was 56.4 (range, 28-71) years (Table 3).

The mean inpatient stay was 4.1 (range, $2-7$ ) days. Three patients required an additional split-skin graft to cover the unburied penile shaft in addition to undergoing the scrotectomy procedure added more length to the penis. One patient developed a superficial wound dehiscence (ClavienDindo grade II). This healed with wound care and regular dressings and did not require further surgery. Two patients had mild residual penile lymphoedema which was managed conservatively with penile compression hosiery, elevation, physiotherapy and daily massage.

No recurrence of the scrotal lymphoedema has occurred at follow up for any of the patients to date. Regression of symptoms and improvement of clinical complaints was 
Table 3 Patient demographics and pre, intra and post-operative parameters

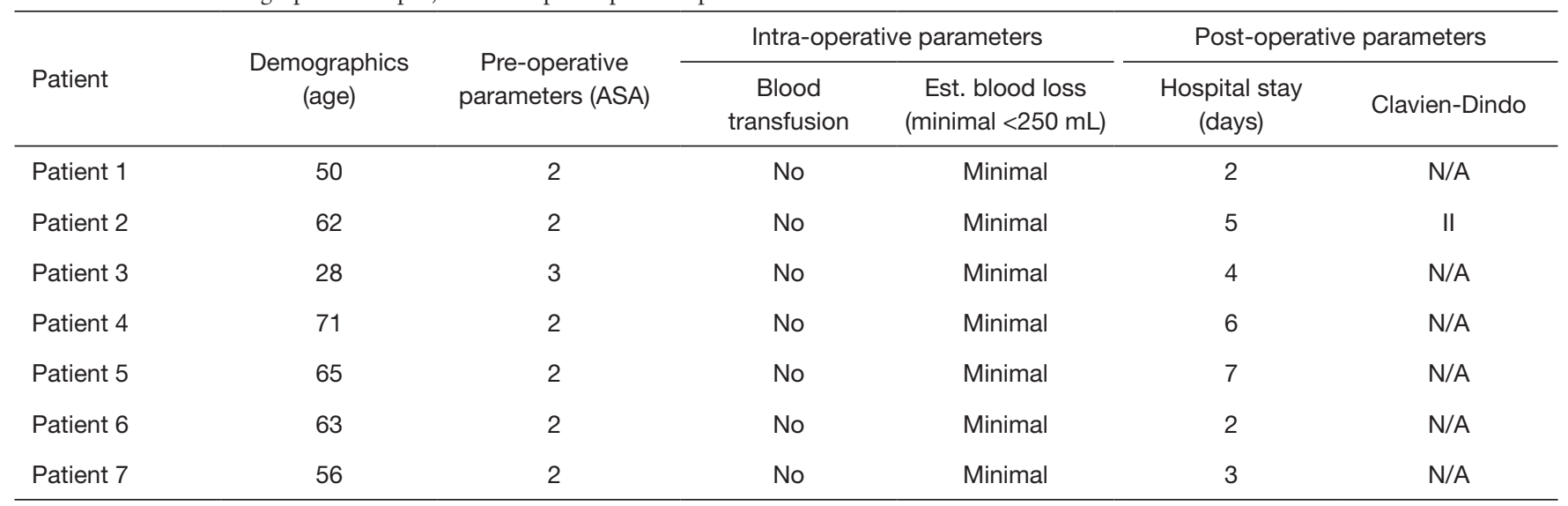

ASA, The American Society of Anesthesiologists; N/A, not applicable.

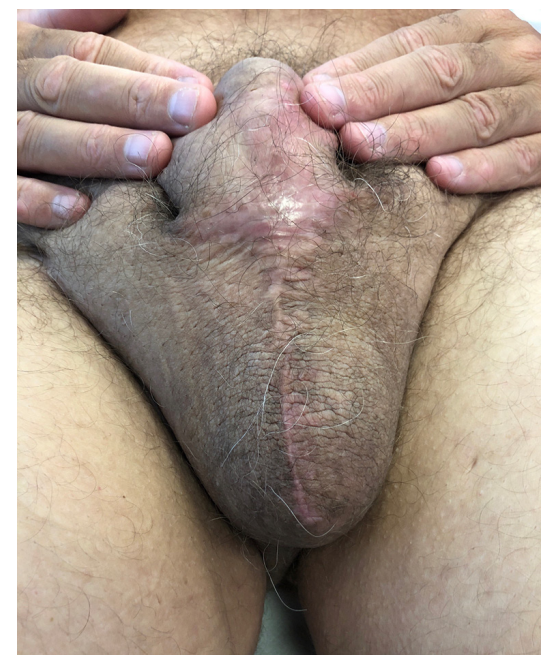

Figure 9 Post-operative appearance at 9 months.

verified at follow-up which ranged from 22 to 43 months with a mean follow-up period of 19 months. Two of the patients in this series died secondary to metastatic penile cancer. One patient developed skin metastasis to his thigh and perineum. However, all of the patients at follow up had a good cosmetic (Figure 9) and good functional outcome from surgery when asked about voiding function (reduced spraying and voiding standing up) and the reduction in the scrotal size (less cumbersome, reduced infections and better hygiene).

Two patients received adjuvant radiotherapy to the inguinal area prior to undergoing a scrotectomy, both of these patients had also undergone pelvic lymphadenectomy. One patient had undergone pelvic lymphadenectomy in addition to inguinal lymph node dissection prior to undergoing the scrotectomy, but did not receive adjuvant radiotherapy. Radiotherapy and or pelvic lymph node dissection did not seem to alter the recovery course and the surgical outcome in these cases. All 3 patients did not develop any post-operative wound complications and are recurrence free to date. All of the patients are standing to void and are able to have penetrative intercourse apart from 1 of the patients who had primary penectomy as the surgical treatment for the primary penile cancer (Table 4).

Histologically in all of the cases the tissue was characterized by marked oedema of the stroma, dilatation of lymphatic vessels and chronic inflammatory infiltrates, lobules of mature adipose tissue separated by fibrous bands. The presence of dilated lymphatic spaces was also noted. Two patients $(28.6 \%)$ had incidental SCC on the histopathological examination of the excised scrotum (Figure 10).

\section{Discussion}

Conventional cancer therapies, including radiotherapy and inguinal lymphadenectomy, have made significant improvements in the treatment outcomes and prognosis of penile cancer patients. Nevertheless, despite various advances in techniques to improve outcomes and decrease morbidity, these interventions can leave patients with significant life changing complications, including lower limb and genital lymphoedema. Scrotal lymphoedema secondary to penile cancer management poses a real challenge to the patient and the medical team involved in their care. Despite meticulous control of the lymphatics during radical inguinal lymphadenectomy, the development 
Table 4 Primary surgery and adjuvant therapy for metastatic penile cancer

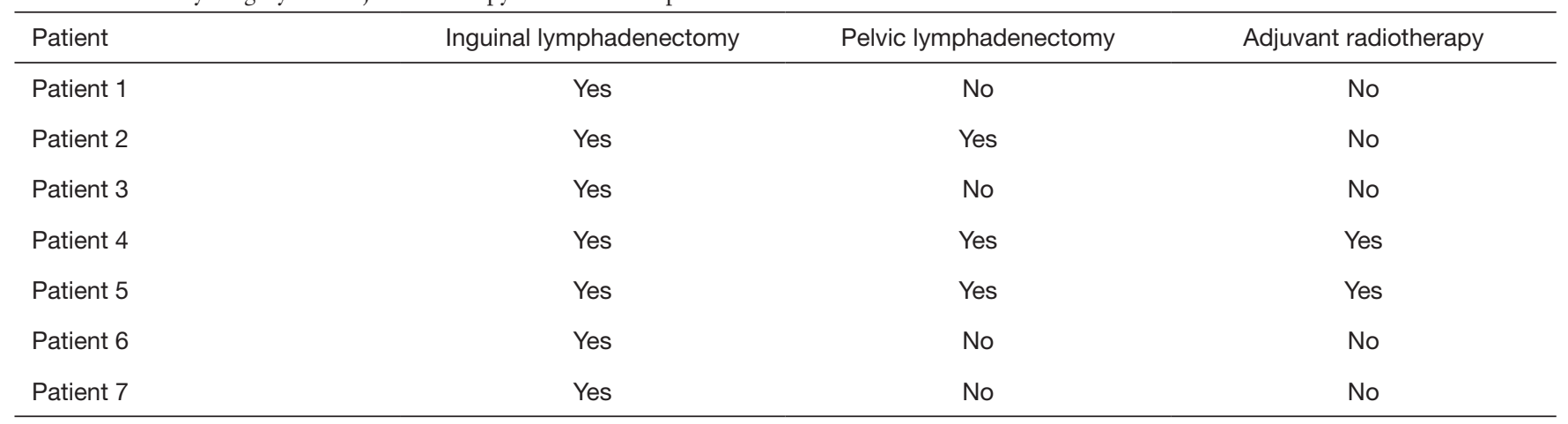

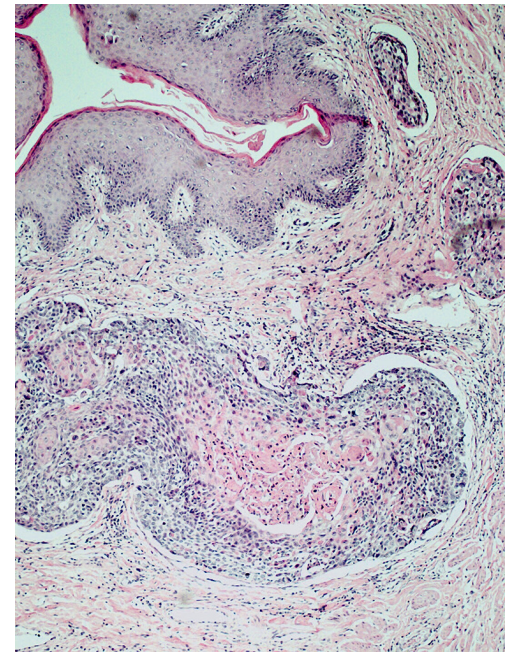

Figure $10 \mathrm{H} \& \mathrm{E}$ section of scrotal skin showing metastatic squamous cell carcinoma with lymphovascular invasion (image published courtesy of Dr. Aiman Haider, Consultant Histopathologist, UCLH, London, UK).

of penoscrotal lymphoedema still occurs. Furthermore, the massive scrotal size often results in burying of the penis which creates difficulty in performing a clinical examination and surveillance of the penis on clinical follow up.

A critical consideration should be given to the management of patient expectations and appropriate counselling prior to their radical inguinal lymphadenectomy. It is important to fully inform patients and explain the potential risk of lymphoedema of the lower limbs and genitalia prior to inguinal or pelvic lymphadenectomy. Based on our series, we advocate that patients with lymphoedema of the external genitalia not responding to conservative methods should be carefully selected and counselled prior to surgical intervention. Patients should initially undergo all conservative therapies and measures prior to surgical management of scrotal lymphoedema. Surgery and genital reconstruction remains the mainstay and the only successful management option to reduce the severe penoscrotal size and the multitude of functional and psychological symptoms that patients present with. Nonetheless, surgery is the last resort. Patients can present with a number of debilitating symptoms which can interfere with daily life activities.

In some cases, the development of acquired lymphangitis circumscripta secondary to obstruction of the lymphatic channels leads to a persistent chronic serous discharge from the scrotal skin. Two recent studies, Tashiro and Zaleska et al. have demonstrated that patients with genital and lower limb lymphoedema have a reduced QOL because of both functional and cosmetic issues $(12,13)$.

Morbidity of conventional inguinal lymphadenectomy has been reported to be as high as $30-50 \%(14,15)$ with a mortality of up to $3 \%$ (16). Complications include wound infection, skin flap necrosis, wound dehiscence, genital and lower limb lymphoedema and lymphocele. In high risk penile cancer, often surgery is followed by radiotherapy and additional pelvic lymphadenectomy with a further increase in the incidence of lymphoedema. A systematic review and meta-analysis of cancer-related secondary lymphoedema in 2010 by Cormier et al. reported increased lymphoedema risk was seen in patients undergoing pelvic lymphadenectomy (22\%) and radiotherapy (31\%) (17). The vast majority of the patients with genital and lower limb lymphoedema following penile cancer treatment are initially managed by conservative measures such as life style changes, compression hosiery and massage techniques combined with avoidance of trauma and infection. Long term compressive hosiery and supportive scrotal garments are sufficient for most cases (4). However, if conservative 
methods fail very few patients are offered a surgical solution due to the risk of wound complications and lack of expertise.

The novel "Batman" incision technique involves an incision shaped like 'Batman' and creates a neo-scrotum using the lateral scrotal flaps, which allows burying of the testicles and suturing of the skin edges such that there is no circumferential junction with the penile base which is brought out through a new circular opening if necessary. Mobilisation of the penis to unbury followed by multiple skin junctions at the base often cause indentations and poor healing. Therefore, keeping the penis away from the cut edges allows healing of the neo-scrotum and the penis can then be brought out from a separate circular incision and the shaft covered with extra-genital split skin graft. The technique allows healthy skin edges to be sutured to create a neo-scrotum thus minimizing the complication rate. In our series, the complication rate was $14.2 \%$ (1/7) indicating that despite radiotherapy and the presence of significant lymphoedema in the tissues, the wide resection margins allow good postoperative wound healing. It is vital to excise all the affected lymphedematous tissue to avoid recurrence of the lymphoedema. The benefit of this technique is that it negates the need for more complex pedicled flaps with their associated risks such as flap ischaemia/necrosis, longer operative times and additional donor site wounds. Another advantage of this technique is that it is easily reproducible and offers good functional and cosmetic outcomes with low recurrence rates. We found adjuvant radiotherapy and previous pelvic lymph node dissection not to be associated with worse outcomes. Modolin et al. reported 1 recurrence of scrotal lymphoedema in a patient who had undergone lymphadenectomy with radiation therapy for penile cancer, from their cohort of 17 patients with genital lymphoedema of the penis and scrotum, regardless of its etiology (2).

In our series, complications were uncommon although mild residual lymphoedema of the penis is reported if a split skin graft on the penis is not used. This can be successfully managed by conservative measures such as compression and massage. Two patients received adjuvant radiotherapy to the inguinal area prior to undergoing a scrotectomy, both of these patients had also undergone pelvic lymphadenectomy. One patient had undergone pelvic lymphadenectomy in addition to inguinal lymph node dissection prior to undergoing scrotectomy, but did not receive adjuvant radiotherapy. The type of surgery for the primary cancer, radiotherapy and or pelvic lymph node dissection did not seem to alter the recovery course and the surgical outcome in these cases. Although, it is well established in the literature that surgery compounded by radiotherapy and additional pelvic lymphadenectomy increase the incidence of lymphoedema, it is difficult to conclude from this cohort whether patients with previous pelvic lymphadenectomy and/or adjuvant radiotherapy are less likely to respond to conservative measure and eventually require surgical management. Garaffa et al. reported that in penoscrotal lymphoedema the scrotum should be treated first as there is often spontaneous resolution of the penile lymphoedema (10). In our series, 2 of the patients with residual penile lymphoedema, resolved following conservative management after few months of the surgery. Additionally, 2 patients were found to have incidental SCC in the scrotum following scrotectomy, which was not detected clinically due to the significant genital lymphoedema. Hence, the importance of close surveillance in penile cancer patients and in particular unburying of the penis in these situations is vital and can be lifesaving. Both of these patients are alive to date.

Various surgical techniques have been advocated in the literature to reduce the lymphoedema size. One technique is the use of fasciocutaneous thigh flaps for coverage of the testes after resection of the scrotal lymphoedema tissue, however, these flaps may alter testicular thermoregulation and lead to testicular atrophy and infertility $(1,7)$. A modified Charles' procedure, which consists of excising the affected skin followed by midline suturing simulating the scrotal raphe is another reported technique (2). Both split and full thickness skin grafts have also been used to cover the site of the defect $(3,5,6)$. The development of new lymphatic drainage channels (lymphangioplasty), is technically challenging and the results have so far been unsatisfactory (18-21). A review by Damstra et al. has also reported that microsurgical lymphatic-venous anastomosis may not be an effective treatment for lymphoedema (22).

Cui et al. reported in a randomised trial comprising 23 patients receiving laparoscopic prophylactic inguinal lymphadenectomy with saphenous vein sparing in one limb and saphenous vein ligation contralaterally, showed saphenous vein preservation does seem to reduce the morbidity of acute genital lymphoedema and lower limb lymphoedema (23). In another study by Dardarian et al. routine preservation of the saphenous vein during inguinal lymphadenectomy for vulvar carcinoma reduced the incidence of cellulitis and chronic lymphoedema (24).

Siegel et al. reported the use of LigaSure in resection of genital lymphoedema in 19 patients to be safe and was 
associated with faster resections, less blood loss per tissue resected, and a lower rate of recurrence (25).

The current evidence in the literature on the management of this debilitating problem is sparse and mainly based on case reports and small case series. Our literature review found no previous studies or case series on the management of penoscrotal lymphoedema following penile cancer therapy.

The limitations of our study are the retrospective nature and the small number of patients included. Due to the retrospective nature of the study, we were unable to use validated QOL improvement tools to objectively measure any changes. One previous study used the Glasgow Benefit Inventory questionnaire to objectively measure improvement in general functioning and physical health (11).

\section{Conclusions}

Genital lymphoedema is an uncommon side effect of penile cancer therapy. Patients can present with debilitating complications and the aim is to restore these. If managed appropriately and in a timely fashion, most patients can be managed by conservative measures. In more severe cases where patients fail conservative treatments, subsequent surgical intervention in appropriately selected and counselled patients is occasionally required as a last resort.

\section{Acknowledgments}

A Muneer is supported by the NIHR Biomedical Research Centre University College London Hospital. This study was supported by the NIHR Biomedical Research Centre University College London Hospital.

\section{Footnote}

Conflicts of Interest: The authors have no conflicts of interest to declare.

Ethical Statement: The authors are accountable for all aspects of the work in ensuring that questions related to the accuracy or integrity of any part of the work are appropriately investigated and resolved. This is a retrospective study. This was not a research and was a departmental audit. As a result, no ethical board review was required. The treatment was in line with our institutional guidelines and approved as part of the MDT process. All data were pseudo-anonymised. All participants gave full informed consent for the photographs to be used in publications.

\section{References}

1. Halperin TJ, Salvin SA, Olumi AF, et al. Surgical management of scrotal lymphedema using local flaps. Ann Plast Surg 2007;59:67-72; discussion 72.

2. Modolin M, Mitre AI, da Silva JC, et al. Surgical treatment of lymphedema of the penis and scrotum. Clinics (Sao Paulo) 2006;61:289-94.

3. Morey AF, Meng MV, McAninch JW. Skin graft reconstruction of chronic genital lymphedema. Urology 1997;50:423-6.

4. McDougal WS. Lymphedema of the external genitalia. J Urol 2003;170:711-6.

5. Lucas JW, Lester KM, Chen A, et al. Scrotal reconstruction and testicular prosthetics. Transl Androl Urol 2017;6:710-21.

6. Chen ML, Odom B, Santucci RA. Surgical management of genitoperineal hidradenitis suppurativa in men. Urology 2014;83:1412-7.

7. Champaneria MC, Workman A, Kao H, et al. Reconstruction of massive localised lymphoedema of the scrotum with a novel fasciocutaneous flap: A rare case presentation and a review of the literature. J Plast Reconstr Aesthet Surg 2013;66:281-6.

8. Salako AA, Olabanji JK, Oladele AO, et al. Surgical Reconstruction of Giant Penoscrotal Lymphedema in SubSaharan Africa. Urology 2018;112:181-5.

9. Machol JA, Langenstroer P, Sanger JR. Surgical reduction of scrotal massive localized lymphedema (MLL) in obesity. J Plast Reconstr Aesthet Surg 2014;67:1719-25.

10. Garaffa G, Christopher N, Ralph DJ. The management of genital lymphoedema. BJU Int 2008;102:480-4.

11. Torio-Padron N, Stark GB, Földi E, et al. Treatment of male genital lymphedema: an integrated concept. J Plast Reconstr Aesthet Surg 2015;68:262-8.

12. Tashiro K, Yamashita S, Saito T, et al. Proximal and distal patterns: different spreading patterns of indocyanine green lymphography in secondary lower extremity lymphedema. J Plast Reconstr Aesthet Surg 2016; 69:368-75.

13. Zaleska M, Olszewski WL, Durlik M. The effectiveness of intermittent pneumatic compression in long-term therapy of lymphedema of lower limbs. Lymphat Res Biol 2014;12:103-9.

14. Bevan-Thomas R, Slaton JW, Pettaway CA. Contemporary morbidity from lymphadenectomy for penile squamous cell 
carcinoma: the MD Anderson Cancer Center Experience. J Urol 2002;167:1638-42.

15. Protzel C, Alcaraz A, Horenblas S, et al. Lymphadenectomy in the surgical management of penile cancer. Eur Urol 2009;55:1075-88.

16. Colberg JW, Andriole GL, Catalona WJ. Longterm follow-up of men undergoing modified inguinal lymphadenectomy for carcinoma of the penis. Br J Urol 1997;79:54-7.

17. Cormier JN, Askew RL, Mungovan KS, et al. Lymphedema beyond breast cancer: a systematic review and meta-analysis of cancer-related secondary lymphedema. Cancer 2010;116:5138-49.

18. Gillies H, Fraser FR. Treatment of lymphoedema by plastic operation. BMJ 1935;1:96-8.

19. Goldsmith HS, De Los Santos R. Omental transposition in primary lymphoedema. Surg Gynecol Obstet 1967;125:607-10.

20. McDonald DF, Huggins C. Surgical treatment of elephantiasis. J Urol 1950;63:187-90.

Cite this article as: Alnajjar HM, Castiglione F, Ahmed K, Haider A, Nigam R, Muneer A. A novel 'Batman' scrotectomy technique for the management of scrotal lymphoedema following treatment for penile cancer. Transl Androl Urol 2019;8(5):448-456. doi: 10.21037/tau.2019.09.04
21. Nielubowicz J, Olsewski W, Sokolowski J. Surgical lympho-venous shunts. J Cardiovasc Surg (Torino) 1968;9:262-7.

22. Damstra RJ, Voesten HG, van Schelven WD, et al. Lymphatic venous anastomosis (LVA) for treatment of secondary arm lymphedema. A prospective study of 11 LVA procedures in 10 patients with breast cancer related lymphedema and a critical review of the literature. Breast Cancer Res Treat 2009;113:199-206.

23. Cui Y, Chen H, Liu L, et al. Saphenous vein sparing during laparoscopic bilateral inguinal lymphadenectomy for penile carcinoma patients. Int Urol Nephrol 2016;48:363-6.

24. Dardarian TS, Gray HJ, Morgan MA, et al. Saphenous vein sparing during inguinal lymphadenectomy to reduce morbidity in patients with vulvar carcinoma. Gynecol Oncol 2006;101:140-2.

25. Siegel JA, Zhao L, Tachibana I, et al. Rapid excision of massive localized lymphedema of the male genitalia with vessel sealing device. Can J Urol 2016;23:8291-5. 